\title{
Flow of Hagedorn singularities and phase transitions in large $N$ gauge theories
}

\author{
Aleksey Cherman, ${ }^{1, *}$ Syo Kamata, ${ }^{2,3, \dagger}$ Thomas Schäfer@, ${ }^{3, \$}$ and Mithat Ünsal ${ }^{3, \S}$ \\ ${ }^{1}$ School of Physics and Astronomy, University of Minnesota Minneapolis, Minnesota 55455, USA \\ ${ }^{2}$ College of Physics and Communication Electronics, Jiangxi Normal University, \\ Nanchang 330022, China \\ ${ }^{3}$ Department of Physics, North Carolina State University, Raleigh, North Carolina 27695, USA
}

(Received 17 November 2019; published 15 January 2020)

\begin{abstract}
We investigate the singularity structure of the $(-1)^{F}$ graded partition function in QCD with $n_{f} \geq 1$ massive adjoint fermions in the large- $N$ limit. Here, $F$ is fermion number and $N$ is the number of colors. The large $N$ partition function is made reliably calculable by taking space to be a small three-sphere $S^{3}$. Singularities in the graded partition function are related to phase transitions and to Hagedorn behavior in the $(-1)^{F}$-graded density of states. We study the flow of the singularities in the complex "inverse temperature" $\beta$ plane as a function of the quark mass. This analysis is a generalization of the Lee-YangFisher-type analysis for a theory which is always in the thermodynamic limit thanks to the large $N$ limit. We identify two distinct mechanisms for the appearance of physical Hagedorn singularities and centersymmetry changing phase transitions at real positive $\beta$, inflow of singularities from the $\beta=0$ point, and collisions of complex conjugate pairs of singularities.
\end{abstract}

DOI: $10.1103 /$ PhysRevD.101.014012

\section{INTRODUCTION}

Singularities of partition functions play an important role in the study of phase transitions. Important examples that have been discussed in the literature are Lee-Yang zeros, Fisher zeros and Hagedorn singularities. Lee and Yang studied zeros of the partition function of a finite system in the presence of an external field [1]. The nature of phase transitions is controlled by the motion of singularities as the system approaches the thermodynamic limit, along with the limiting distribution of the Lee-Yang zeros. Fisher studied zeros of the partition function as a function of a complex temperature or interaction parameter [2]. Hagedorn singularities are generally a signature of confinement in large $N$ gauge theories, see e.g., [3-5]. They are produced by exponential growth in the number of states as a function of energy, which is believed to be related to the excitation spectrum of confining strings. The existence of these singularities in the thermal partition function is tied to

\footnotetext{
*acherman@umn.edu

†skamata11phys@gmail.com

tmschaef@ncsu.edu

§unsal.mithat@gmail.com
}

Published by the American Physical Society under the terms of the Creative Commons Attribution 4.0 International license. Further distribution of this work must maintain attribution to the author(s) and the published article's title, journal citation, and DOI. Funded by SCOAP ${ }^{3}$. the inevitability of a deconfinement phase transition as a function of temperature in e.g., large $N$ Yang-Mills (YM) theory [6].

In this work we investigate the motion of partition function singularities of large $N$ gauge theories on compactified spaces. There are several novel aspects compared to the works of Lee, Yang and Fisher. First, gauge theories with a large number of colors $N$ can have phase transitions even in a finite volume, because the large $N$ limit is basically a thermodynamic limit [7]. In the present work we take advantage of this fact by using a finite spatial volume to study phase transitions at weak gauge coupling. We work in Euclidean spacetime signature, take space to be a threesphere $S^{3}$ with radius $R$, and assume that Euclidean time is a circle $S^{1}$ of circumference $\beta$. For an asymptotically free theory with strong scale $\Lambda$, the limit $R \Lambda \ll 1$ is a weakcoupling limit. Gauge theories in this limit were first studied in Refs. [8-10].

Our focus will be on the singularity structure of YangMills theory with $n_{f}$ flavors of Majorana adjoint fermions, QCD(adj), for different values of the fermion mass $m$. We take $1 \leq n_{f} \leq 5$, where the upper bound comes from the requirement of asymptotic freedom, and assume that all of the quarks have a common mass $m$. We will consider the graded partition function

$$
\tilde{Z}(\beta)=\operatorname{tr}\left[e^{-\beta H}(-1)^{F}\right],
$$

where $H$ is the Hamiltonian and $F$ is fermion number, and $\beta$ is the circle size. Adjoint QCD is interesting for many 
reasons, and has been studied extensively in recent years, see e.g., [11-29]. Crucially, it has a $\mathbb{Z}_{N}$ center symmetry $[30,31]$. The existence of this symmetry makes the transition from confinement to deconfinement a sharply defined notion. The deconfinement transition is associated with spontaneous breaking of $\mathbb{Z}_{N}$ center symmetry.

A number of aspects of the $m$ dependence of large $N$ adjoint QCD on $S^{3} \times S^{1}$ was studied by Myers and Hollowood [32], with a focus on the behavior of center symmetry. Our analysis, which agrees with theirs on all points of overlap, instead focuses on the behavior of flow of Hagedorn singularities in the graded partition function. The leading Hagedorn singularities are associated with an inverse "temperature" scale $\beta_{H}$. In general, $\beta_{d}>\beta_{H}$ $[10,33]$, but in the $R \Lambda \rightarrow 0$ regime on which we focus, $\beta_{d} \rightarrow \beta_{H}$. So studying the Hagedorn singularity structure of the partition function is another way to study the fate of color confinement in the theory.

The two features of adjoint QCD of prime importance for our work are

(1) When $m \rightarrow \infty$, the fermions decouple, and $\tilde{Z}(\beta)$ reduces to the thermal partition function $Z(\beta)=$ tre $e^{-\beta H}$ of pure Yang-Mills theory without matter, $n_{f}=0$ :

$$
Z_{\mathrm{YM}}(\beta)=\tilde{Z}(\beta, m=\infty) .
$$

Then there is a thermal deconfinement transition at $\beta \sim R$ when $R \Lambda \ll 1$ [8-10], and $\beta$ can be interpreted as inverse temperature.

(2) When $m \rightarrow 0$, the bosonic and fermionic spectrum of large $N$ adjoint QCD becomes highly correlated in such a way that there are no phase transition in $\tilde{Z}$ as a function of $\beta$ [34-36]. The compactification in this limit is nonthermal and $\beta$ is interpreted as circle size.

These two features are related to Hagedorn singularities. To discuss these singularities, we define the parameter $q=e^{-\beta / R}$. The large $N$ partition function $Z$ of pure YangMills has infinitely many poles $q_{i}$ in the domain $q \in[0,1]$ [8-10]. The inverse Laplace transform of $Z$ is the density of states $\rho(E)$, and each pole $q_{i}=e^{-\beta_{i} / R}$ in $Z$ corresponds to an exponential "Hagedorn" factor $\rho(E) \sim e^{\beta_{i} E}$ in a large- $E$ expansion of $\rho(E)$. This behavior implies that pure YM theory must have a phase transition to a deconfined phase at some $\beta \geq \max \left(\beta_{i}\right)$.

The behavior of the graded partition function $\tilde{Z}$ of adjoint QCD with $m=0$ is quite different. To see why, note that $n_{f}=1 \mathrm{QCD}(\operatorname{adj})$ is $\mathcal{N}=1$ supersymmetric (SUSY) YangMills theory, and the supersymmetry of the theory on $\mathbb{R}^{4}$ suggests that bosonic and fermionic excitations should

\footnotetext{
${ }^{1}$ We put the word temperature in quotes because we are discussing the $(-1)^{F}$-graded partition function, rather than the thermal partition function.
}

cancel in the $(-1)^{F}$ graded partition function. Despite the subtlety that the supersymmetry of pure $\mathcal{N}=1 \mathrm{SYM}$ is broken by coupling to the curvature of $S^{3}$, the remaining cancellations remain strong enough that $\tilde{Z}(\beta)$ has no singularities for $q \in[0,1]$. The graded partition function is associated to a graded density of states, $\tilde{\rho}(E)=$ $\rho_{\mathcal{B}}(E)-\rho_{\mathcal{F}}(E)$. Despite the fact that both $\rho_{\mathcal{B}}(E)$ and $\rho_{\mathcal{F}}(E)$ exhibit Hagedorn growth, $\tilde{\rho}(E)$ does not. The absence of Hagedorn behavior in $\tilde{\rho}(E)$ generalizes to QCD(adj) with $n_{f} \geq 2$, implying that there are strong Bose-Fermi cancellations even without supersymmetry [34-36]. In particular, QCD(adj) with $n_{f} \geq 1$ massless adjoint fermions has no phase transition on a small $S^{3} \times S^{1}$ for any $\beta \in[0, \infty)[35,37]$.

This leads to an interesting issue which we study in this paper. Consider turning on a small mass for the adjoint fermion, $m>0$. Since the YM thermal partition function $Z_{\mathrm{YM}}(\beta)=\tilde{Z}(\beta, m=\infty)$ has infinitely many singularities in the physical domain $q \in[0,1]$, but $\tilde{Z}(\beta, m=0)$ does not have any singularities in the physical domain, the positive real singularities must start to appear at some finite value $m=m_{c}$. In this work we study the mechanism by which this happens. In analogy with the Lee-Yang-Fisher analysis, we show that the flow of complex Hagedorn singularities $q=e^{-\beta / R+i t} \in \mathbb{C}$ is associated with center-symmetry changing phase transitions in large $N$ gauge theories on $S^{3}$. There are two basic scenarios that lead to the appearance of real singularities, the collision of complex conjugate singularities, or the inflow of singularities from the endpoint $q=1$.

\section{SETTING AND GRADED PARTITION FUNCTION}

We now describe the setting for our analysis in more detail. When Yang-Mills theories are placed on a spatial $S^{3}$, the gauge fields and fermions pick up effective masses due to the curvature of $S^{3}[10]$. When $R \Lambda \ll 1$, the curvature is large, so the effective coupling $\lambda(1 / R)$ becomes small, and we can integrate out all of the nonzero modes of the gauge fields at one loop, which amounts to working at $\lambda=0$. But the part of the path integral associated with the holonomy

$$
\alpha:=\frac{1}{\operatorname{Vol}(\mathcal{M})} \int_{S^{3} \times S^{1}} A_{0},
$$

must be treated exactly, and the path integral reduces to a matrix integral over $\alpha$. One can diagonalize $\alpha$ using a global gauge transformation,

$$
\alpha_{\text {diag }}=\beta^{-1} \operatorname{diag}\left(\theta_{1}, \ldots, \theta_{N}\right) .
$$

and then

$$
\tilde{Z}=\int D \alpha_{\mathrm{diag}} e^{-S_{\mathrm{eff}}\left[\alpha_{\mathrm{diag}}\right]}
$$


where $S_{\text {eff }}\left[\alpha_{\text {diag }}\right]$ includes a contribution from the Haar measure. The calculation of the one-loop effective action $S_{\text {eff }}$ is described in [10,32]. At large $N$, the difference between $S U(N)$ and $U(N)$ gauge groups becomes negligible for the purposes of this paper, but the effective action of the $U(N)$ theory is simpler. So we will work with $S_{\text {eff }}$ for $U(N)$ gauge theory. On small $S^{3}$, this effective action takes the form $[10,32]$

$$
S_{\text {eff }}(\alpha)=\sum_{n=1}^{\infty} \frac{f_{n}(q, m)}{n}\left|\operatorname{Tr}\left(P^{n}\right)\right|^{2},
$$

where $P=e^{i \beta \alpha}$ is the Polyakov line, and

$$
f_{n}\left(q^{n}, m R\right):=1-z_{v}\left(q^{n}\right)+n_{f} z_{f}\left(q^{n}, m R\right),
$$

Here, $z_{v}$ and $z_{f}$ are single particle partition functions for vector fields and fermions, and

$$
z_{v}(q)=2 \sum_{\ell=1}^{\infty} \ell(\ell+2) e^{-\beta(\ell+1) / R}=\frac{6 q^{2}-2 q^{3}}{(1-q)^{3}},
$$

When $m=0, z_{f}$ takes the simple form

$$
z_{f}(q)=2 \sum_{\ell=1}^{\infty} \ell(\ell+1) e^{-\beta(\ell+1 / 2) / R}=\frac{4 q^{3 / 2}}{(1-q)^{3}} .
$$

But when $m R>0$, it is simpler to write $z_{f}$ as a function of $\beta / R=-\log q$ in the form [32]

$$
\begin{aligned}
z_{f}\left(\beta / R, m_{f} R\right) \\
=2 \sum_{\ell=1}^{\infty} \ell(\ell+1) e^{-\beta \sqrt{(\ell+1 / 2)^{2}+m_{f}^{2} R^{2}} / R} \\
=\frac{2 m_{f}^{2} R^{3}}{\beta} K_{2}\left(m_{f} \beta\right)-\frac{m_{f} R}{2} K_{1}\left(m_{f} \beta\right) \\
\quad+4 \int_{m_{f} R}^{\infty} d x \frac{x^{2}+1 / 4}{e^{2 \pi x}+1} \sin \left(\beta \sqrt{x^{2}-m_{f}^{2} R^{2}} / R^{2}\right) .
\end{aligned}
$$

It is easy to verify that when $q \rightarrow 0(\beta \rightarrow \infty)$, all of the coefficients $f_{n}$ are positive. This implies that $S_{\text {eff }}$ is minimized if all moments $\rho_{n}=\operatorname{Tr}\left(P^{n}\right)(n \geq 1)$ are zero, and the theory is in the center-symmetric (that is, confining) phase. The partition function is obtained by integrating over the Gaussian modes $\rho_{n}$, which gives

$$
\tilde{Z}(q)=\prod_{n=1}^{\infty} \frac{1}{f_{n}\left(q^{n}, m R\right)} .
$$

This expression is physically valid so long as all $f_{n}$ are positive, which is in general only true for some range of values of $q$ in the real interval $\left[0, q_{c}\right], q_{c} \leq 1$, with $q_{c}=$ $q_{c}(m)$. However, we find it useful to analytically continue
$\tilde{Z}$ to a function of $q$ within the unit disk $|q|<1$ in the complex $q$ plane, and in the rest of this paper $\tilde{Z}$ will denote this analytically continued quantity.

It will be useful to compare the graded partition function $\tilde{Z}$ to the standard thermal partition function $Z$. On small $S^{3} \times S^{1}$, the thermal partition function is obtained by replacing

$$
f_{n}\left(q^{n}, m R\right) \rightarrow b_{n}\left(q^{n}, m R\right)
$$

where

$$
b_{n}\left(q^{n}, m R\right)=1-z_{v}\left(q^{n}\right)+(-1)^{n} n_{f} z_{f}\left(q^{n}, m R\right),
$$

so that

$$
Z=\prod_{n=1}^{\infty} \frac{1}{b_{n}\left(q^{n}, m R\right)}
$$

It is known since Refs. [10,32] that $Z$ has infinitely many poles in the interval $q \in[0,1]$ for any $n_{f} \geq 0$, corresponding to real positive solutions of $b_{n}\left(q^{n}, m R\right)=0$ for $n \geq 1$ in this range. As discussed in [35,38], there are also infinitely many complex poles within the unit disc $|q| \leq 1$, where $q \in \mathbb{C}$, but for the Hagedorn growth of the spectral density only the poles $q \in[0,1]$ are relevant.

\section{HAGEDORN TRANS-SERIES}

To understand the connection between singularities in the partition function and Hagedorn behavior of the density of states, let us write the $(-1)^{F}$ density of states $\tilde{\rho}(E)=$ $\rho_{\text {bosonic }}(E)-\rho_{\text {fermionic }}(E)$ as a trans-series expansion in variable $x=1 /(E R)$ around the point $x=0$. A natural ansatz for the form of $\tilde{\rho}(E)$ is

$$
\begin{aligned}
R^{-1} \tilde{\rho}(E)= & e^{\beta_{1} / x} \sum_{n=0}^{\infty} a_{1, n} x^{n}+e^{\beta_{2} / x} \sum_{n=0}^{\infty} a_{2, n} x^{n}+\cdots \\
& +\frac{1}{2}\left(e^{\gamma_{1} / x}+e^{\gamma_{1}^{*} / x}\right) \sum_{n=0}^{\infty} c_{1, n} x^{n} \\
& +\frac{i}{2}\left(e^{\delta_{1} / x}-e^{\delta_{1}^{*} / x}\right) \sum_{n=0}^{\infty} d_{1, n} x^{n}+\cdots
\end{aligned}
$$

where $a_{n, k}, c_{n, k}, d_{n, k}, \beta_{n}, \gamma_{n}, \delta_{n}$ are dimensionless parameters, $\beta_{n} \in \mathbb{R}$ with $\beta_{1}>\beta_{2}>\cdots$, and $\gamma_{n} \in \mathbb{C}$ with $\operatorname{Re} \gamma_{1}>$ $\operatorname{Re} \gamma_{2}>\cdots$ and $\operatorname{Im} \gamma_{n} \neq 0$ for all $n$ (and similarly for $\delta_{n}$ ). The inverse Hagedorn temperature scales are $\beta_{H, n}=\beta_{n} R$, which are physical when $\beta_{n}>0$.

This is only the simplest ansatz for $\tilde{\rho}(E)$ which incorporates the phenomena we want to describe. Physically, however, we expect the trans-series representation of $\tilde{\rho}(E)$ to depend on more transmononomials of $x$, such as $\log (x)$ and $x^{b}$ with $b \notin \mathbb{N}$. Determining the complete trans-series 
representation of $\rho(E)$ is a currently open problem even in the simplest cases such as $m=0$ and $m=\infty$, where $\tilde{Z}$ (and also $Z$ ) can be written as products of elliptic functions $[39,40]$. It is likely that this problem can be solved using the methods of Ref. [41], and it would be interesting to do so in future work.

To see how this ansatz for the density of states relates to the partition function, note that we can write

$$
\tilde{Z}(\beta)=\operatorname{tr}(-1)^{F} e^{-\beta H}=\int_{E_{0}}^{\infty} d E \tilde{\rho}(E) e^{-\beta E} .
$$

where $E_{0}=c / R$ with $c=3 / 2$. The cutoff on the energy integral at $E_{0}$ takes into account that the spectrum of $4 \mathrm{~d}$ adjoint $U(N)$ QCD on small $S^{3}$ is gapped with a gap that interpolates between $E_{0}$ at $m=0$ and $4 / 3 E_{0}$ at $m=\infty$. So $\tilde{\rho}(E)$ must always vanish for $E<E_{0}$. Evaluating the integral gives

$$
\begin{aligned}
\tilde{Z}(\beta)= & \frac{1}{\beta-\beta_{1}}\left\{a_{1,0}+\left[c a_{1,0}-a_{1,1}\left(\gamma_{E}+\log \left[c\left(\beta-\beta_{1}\right)\right]\right)\right.\right. \\
& \left.+\sum_{n=2}^{\infty} \frac{a_{1, n}}{c(n-1)}\right]\left(\beta-\beta_{1}\right) \\
& +\left[\frac{c^{2}}{2} a_{1,0}+c a_{1,1}+a_{1,2}\left(\gamma_{E}-1+\log \left[c\left(\beta-\beta_{1}\right)\right]\right)\right. \\
& \left.\left.+\sum_{n=3}^{\infty} \frac{a_{n}}{c(n-1)}\right]\left(\beta-\beta_{1}\right)^{2}\right\}+\ldots \\
& +\frac{1}{\beta-\beta_{2}}\left[a_{2,0}+\ldots\right]+\ldots
\end{aligned}
$$

This expression illustrates that exponentially-growing terms in $\tilde{\rho}(E)$ generate singularities in $\tilde{Z}(\beta)$. The coefficients $\beta_{n}$, $a_{n, k}, \gamma_{n}, c_{n, k}, \delta_{n}, d_{n, k}$ all depend on the quark mass $m$.

We note that the thermal partition function $Z(\beta)$ and the thermal (not graded) density of states $\rho(E)$ can be written in the same form, but of course the numerical values of the parameters entering the thermal density of states transseries are different from the $(-1)^{F}$-graded one.

\section{FLOW OF HAGEDORN SINGULARITIES}

Let us now work out the flow of the Hagedorn parameters $\beta_{n}$ and $\gamma_{n}$ as function of the quark mass $m$. One can easily verify that $f_{n}\left(q^{n}, m=0\right)$ is positive for all $q \in[0,1]$, so there are no poles of $\tilde{Z}(q)$ for $q \in[0,1]$ [35]. Correspondingly, there is no Hagedorn growth in the graded density of states, $\tilde{\rho}(E)$. This means that $\beta_{n}<0$ for all $n$ at $m=0$, but it does not constrain $\gamma_{n}$. Physically, these remarks imply that the infinitely large number of Hagedorn-growing terms in both $\rho_{\mathcal{B}}(E)$ and $\rho_{\mathcal{F}}(E)$ cancel against each other, leaving a function with subexponential growth in $E$. The main point of $[34,36]$ is that this is not an artifact of working at small $R \Lambda$. It is related to the fact that it enjoys large $N$ volume independence [11,21-23,27-29, 42-44]. In infinite volume, on $\mathbb{R}^{3} \times S^{1}$ and $N=\infty$, adjoint QCD endowed with periodic boundary conditions does not have any phase transitions. This implies that the physical gauge-invariant bosonic and fermionic excitations of adjoint QCD are very tightly correlated despite the manifest absence of supersymmetry in adjoint QCD for $n_{f}>1$.

Now suppose $m \neq 0$. As mentioned in the Introduction, at $m=\infty$ the fermions decouple from the spectrum of the theory, so that

$$
\lim _{m \rightarrow \infty} \tilde{Z}(q, m)=Z_{\mathrm{YM}}(q) .
$$

Therefore, $\tilde{Z}(q, 0)$ is free of poles $q \in[0,1]$, but $\tilde{Z}(q, \infty)$ has infinitely many poles. So when $m \rightarrow \infty, \beta_{1}$ along with an infinite subset of the $\beta_{n}$ coefficients are positive. In the free limit, the Hagedorn temperature $\beta_{1}$ corresponds to a center-symmetry changing phase transition.

Combining these observations, we see that as $m$ is varied in $[0, \infty)$ a pole on the real interval $[0,1]$ has to appear at some critical mass $m_{c}$. Tracking the motion of the Hagedorn singularities is a difficult task in general, but at small $R \Lambda$ it can be done explicitly thanks to the fact that the theory is weakly coupled.

We find two distinct types of Hagedorn singularity flow.
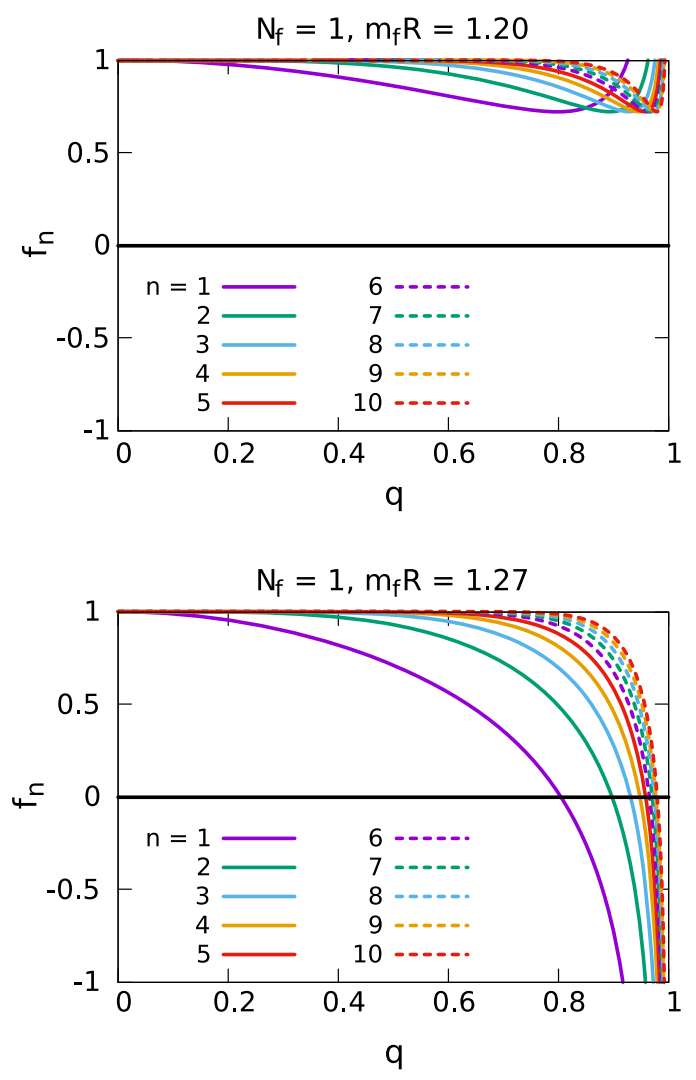

FIG. 1. Plots of $f_{n}(q)$ as a function of real $q \in[0,1]$ for $n_{f}=1$. The top panel shows $m_{f} R=1.20$, below the critical mass. The bottom panel shows $m_{f} R=1.27$, above the critical mass. 


\section{ADJOINT QCD WITH $n_{f}=1$}

This is the only value of $n_{f}$ at which the $m=0$ limit of the theory has $\mathcal{N}=1$ supersymmetry on $\mathbb{R}^{3} \times S^{1}$. Perhaps not coincidentally, we find that the flow of the Hagedorn singularities at $n_{f}=1$ is quite different from the flow at higher $n_{f}$. For $n_{f}=1$, we show the functions $f_{n}(q)$ along the real interval $q \in[0,1]$ in Fig. 1, while the singularities in the complex $q$-plane are shown in Fig. 2. Both plots show the behavior just below and just above the critical mass $m_{c} R \simeq 1.22$.

One can see that as $m$ increases pass $m_{c}$, all $f_{n}(q)$ change sign for some value of $q$ and stay negative as $q \rightarrow 1$. A related observation is that the zeros in $f_{n}(q)$ appear from $q=1$ limit as $m$ is increased. This is reflected in the complex $q$-plane, where singularities of $\tilde{Z}$ flow in from the point $q=1$ once $m>m_{c}$. The distribution of these singularities approaches the distribution of the singularities of the partition function of pure YM theory (obtained in the limit $m \rightarrow \infty$ ) by moving inward along the real axis. The singularities on $q \in[0,1)$ have an interpretation as terms that scale as $e^{\beta_{n} E}$ with $\beta_{n}>0$, in the graded density of states.

For $m<m_{c}$, the theory preserves the full $\mathbb{Z}_{N}$ centersymmetry at any value of $\beta / R$. For $m>m_{c}$, the $\mathbb{Z}_{N}$ centersymmetry is broken completely-that is, it is broken to $\mathbb{Z}_{1}$.
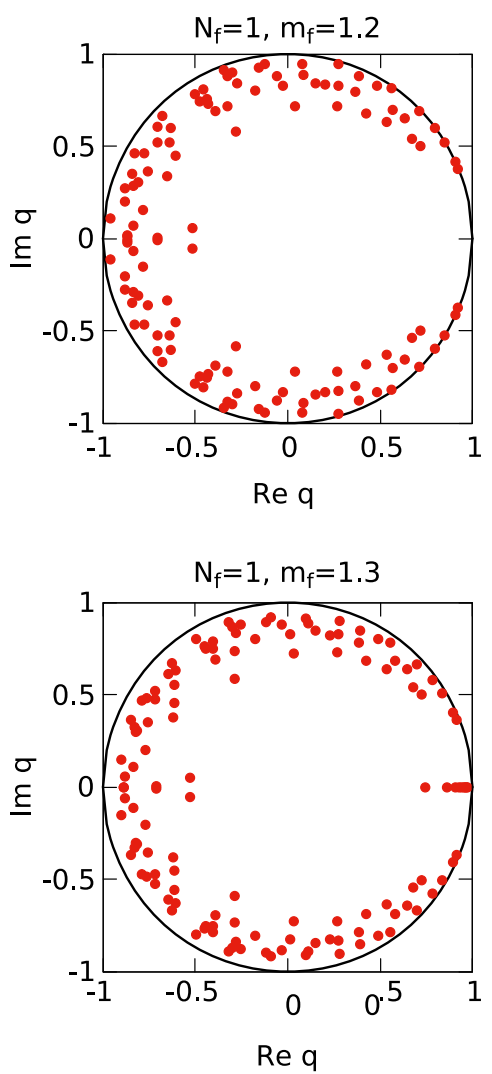

FIG. 2. Complex $q$-plane for $N_{f}=1$ near $m_{f}=m_{f}^{*}$. Top panel: $N_{f}=1, m_{f} R=1.2$. Bottom panel: $N_{f}=1, m_{f} R=1.3$.
However, as $m_{c}$ is lowered from $\infty$ to $m_{c}$, the center-breaking scale of one-flavor adjoint QCD increases smoothly from the deconfinement temperature of pure YM theory, $T_{\mathrm{YM}}$, to $\beta^{-1}=\infty$, connecting a thermal phase transition to a quantum phase transition.

\section{ADJOINT QCD WITH $\boldsymbol{n}_{f} \geq \mathbf{2}$}

We have checked that the behavior for $n_{f}=2$ and higher $n_{f}$ is qualitatively identical, so for simplicity we focus our discussion on the case $n_{f}=2$. The function $f_{n}(q)$ and the singularities in the complex $q$-plane are shown in Figs. 3 and 4. The critical value of $m$ is now $m_{c} R \simeq 3.20$.

When $m<m_{c}$ the functions $f_{n}(q)$ do not cross the $f_{n}=0$ line, but have minima that approach it as $m \rightarrow m_{c}$. This is reflected by complex conjugate pairs of singularities in the $q$-plane that approach, but do not cross, the positive real axis. The theory preserve the full $\mathbb{Z}_{N}$ center-symmetry at any value of $\beta / R$ when $m<m_{c}$. However, when $m>m_{c}$, the pairs of singularities collide and move onto the positive real $q$ axis. This corresponds to a deconfining phase transition, which we now examine.

The first big difference one can see from the plots in Figs. 3,4 is that the $\mathbb{Z}_{N}$ changing phase transition scale is finite if $m$ approaches $m_{c}$ from above. This should be contrasted with what we saw when $n_{f}=1$, when the $\mathbb{Z}_{N}$
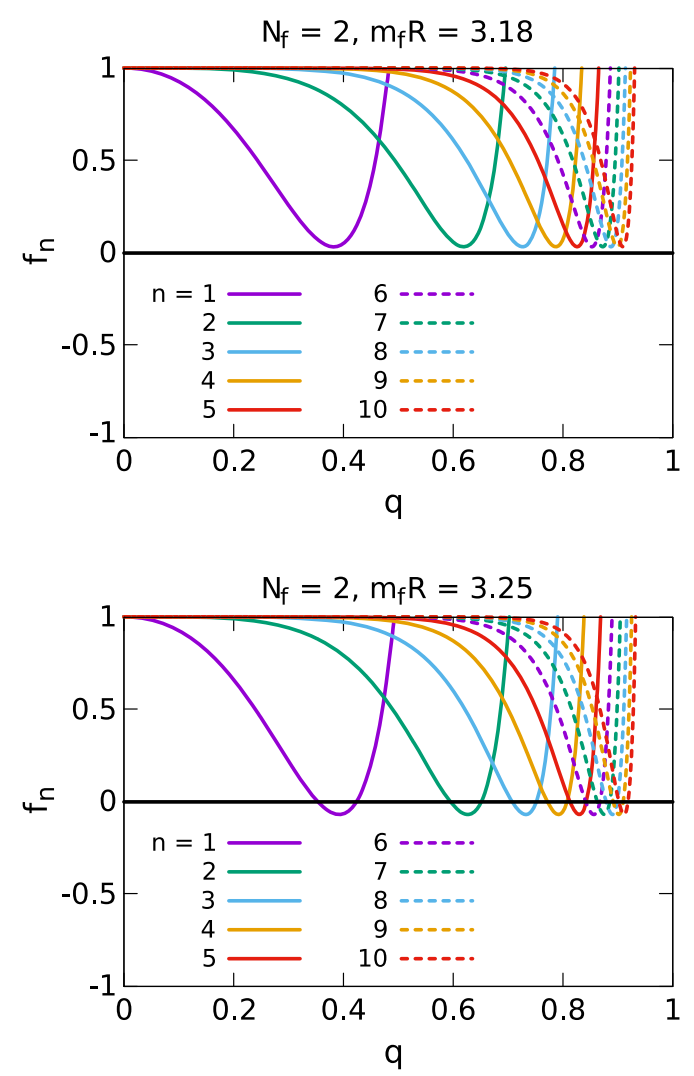

FIG. 3. Plots of $f_{n}(q)$ as a function of real $q \in[0,1]$ for $n_{f}=2$. Top panel: $m_{f} R=3.18$. Bottom panel: $m_{f} R=3.25$. 

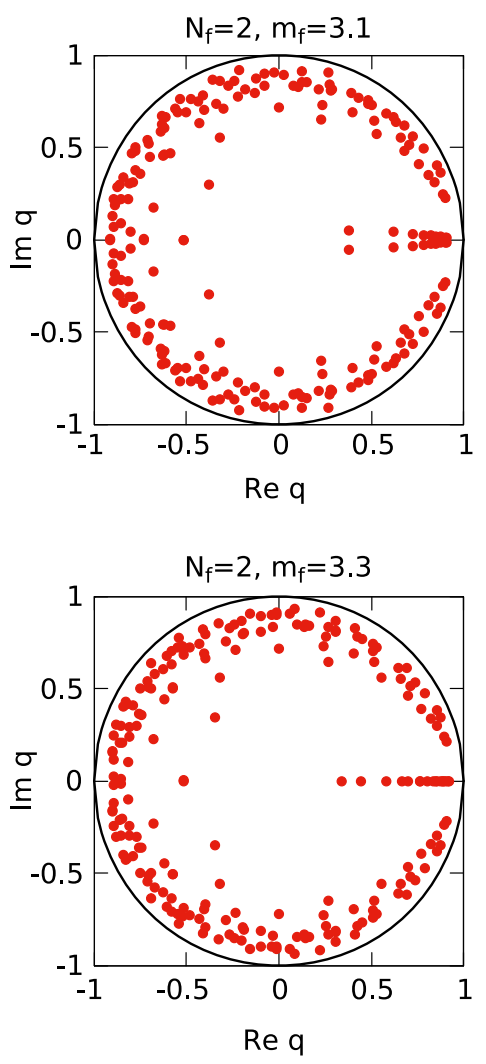

FIG. 4. Complex $q$-plane for $N_{f}=2$ near $m_{f}=m_{f}^{*}$. Top panel: $N_{f}=2, m_{f} R=3.1$. Bottom panel: $N_{f}=2, m_{f} R=3.3$.

changing transition scale $\beta \rightarrow 0$ when $m$ approaches $m_{c}$ from above.

Next, let us understand the properties of the transition in more detail. Suppose that $m \gg m_{c}$ (large mass limit) and lower $\beta / R$ starting from $\beta / R=\infty$ [32]. The theory starts out in the center-symmetric phase. At a critical value of $\beta / R$ there is a phase transition to a phase where $\mathbb{Z}_{N} \rightarrow \mathbb{Z}_{1}$. As $\beta / R$ is reduced further, $f_{1}$ eventually becomes positive, while $f_{n \geq 2}$ are still negative. Hence, the center partially restores to $\mathbb{Z}_{2}$. In fact, as $\beta / R$ is reduced, in the large $N$ limit we observe the pattern

$$
\mathbb{Z}_{N} \rightarrow \mathbb{Z}_{1} \rightarrow \mathbb{Z}_{2} \rightarrow \mathbb{Z}_{3} \rightarrow \mathbb{Z}_{4} \cdots
$$

In the phase where the center-symmetry breaking pattern is $\mathbb{Z}_{N} \rightarrow \mathbb{Z}_{k}$, the gauge structure becomes $[U(N / k)]^{k}$ where eigenvalues of Wilson line split into $k$ bunches each of which has $N / k$ coincident eigenvalues.

An interesting structure appears for $\left(m-m_{c}\right) R=\epsilon$ small and positive. Reducing $\beta / R$, we observe centersymmetry breaking $\mathbb{Z}_{N} \rightarrow \mathbb{Z}_{1}$ at the location where $f_{1}=0$. But $f_{1}$ turns around and becomes positive again while all other $f_{n \geq 2}$ are still positive. Continuing to decrease $\beta, f_{2}$ switches sign, and repeats the same pattern. Hence, there are "pockets" of restored $\mathbb{Z}_{N}$ symmetry, with the pattern

$$
\mathbb{Z}_{N} \rightarrow \mathbb{Z}_{1} \rightarrow \mathbb{Z}_{N} \rightarrow \mathbb{Z}_{2} \rightarrow \mathbb{Z}_{N} \rightarrow \mathbb{Z}_{3} \cdots
$$

As $\epsilon$ is increased, these pockets of full-center restoration close, and the pattern turns into that of Eq. (19).

\section{PHASE TRANSITION AND GRADED DENSITY OF STATES}

In the trans-series discussion, we provided a general form for the Hagedorn trans-series (15). It is instructive to study the phase transition in terms of the graded density of states $\tilde{\rho}(E)$, and to investigate how the trans-series structure for $\tilde{\rho}(E)$ changes as a function of mass parameter. We consider the case $n_{f} \geq 2$. As $m \rightarrow m_{c}$, infinitely many complex conjugate poles coalesce on the real axis $q \in[0,1]$. For $m=m_{c}+0^{+}$, we can enumerate the poles on the positive real axis $\mathbb{R}_{\beta}^{+}$as $\beta_{H_{1}}>\beta_{H_{2}}>\beta_{H_{3}}>\ldots$, where $e^{\beta_{H_{1}} E}$ is the leading Hagedorn growth in the density of states. To get a feeling for the physics, let us study a simplified toy model where the partition function only has the two most dominant poles at $\beta=\beta_{H_{1}}, \beta_{H_{2}}$.

The density of states is the inverse Laplace transform of the partition function. A toy model describing the collision of the two poles is defined by

$$
\tilde{Z}(\beta, m)= \begin{cases}\left(\beta-\beta_{H_{1}}\right)^{-1}\left(\beta-\beta_{H_{2}}\right)^{-1} & m>m_{c} \\ \left(\beta-\beta_{H_{1}}\right)^{-2} & m=m_{c} \\ \left(\beta-\beta_{H}\right)^{-1}\left(\beta-\beta_{H}^{*}\right)^{-1} & m<m_{c}\end{cases}
$$

where $\beta_{H}=\beta_{H_{R}}+i \beta_{H_{I}} \in \mathbb{C}$. When $m$ is large, there are two isolated real poles at $\beta=\beta_{H_{1}}$ and $\beta_{H_{2}}$. But when $m$ is decreased toward $m=m_{c}$ the two isolated real poles merge into a double pole, and move into the complex plane as complex conjugate poles for $m<m_{c}$. The inverse Laplace transform is

$$
\tilde{\rho}(E)= \begin{cases}\frac{e^{\beta_{H_{1}} E}-e^{\beta_{H_{2}} E}}{\beta_{H_{1}}-\beta_{H_{2}}} & m>m_{c} \\ E e^{\beta_{H_{1}} E} & m=m_{c} \\ \beta_{H_{I}}^{-1} e^{\beta_{H_{R}} E} \sin \left(\beta_{H_{I}} E\right) & m<m_{c}\end{cases}
$$

In the regime where the center symmetry is completely broken, the Hagedorn growth $e^{\beta_{H_{1}} E}$ is the dominant contribution to $\tilde{\rho}(E)$. In the domain where center is intact, $\tilde{\rho}(E)$ is oscillatory, consistent with the general trans-series structure (15). The frequency of oscillation is controlled by the imaginary part of the Hagedorn temperature, and in the limit where $\beta_{H_{I}} \rightarrow 0$, the oscillations disappear. Oscillations of the spectral density in the limit $m \rightarrow 0$ were studied in [35]. These authors noted that Bose-Fermi cancellations are responsible for the disappearance of the Hagedorn growth in the graded density of states. This cancellation, unlike supersymmetry, does not take place level-by-level. Instead, the cancellations involve many neighboring levels, in a pattern essentially identical to that 
of "misaligned supersymmetry" in string theory [45-47]. Eventually, once $m>m_{c}$, the Bose-Fermi balance breaks down and Hagedorn growth emerges.

\section{OUTLOOK}

In this work we have studied to motion of Hagedorn singularities in the complexified $q=\exp (-\beta / R)$ plane of large $N$ Yang-Mills theory with $n_{f}$ adjoint fermions compactified on $S^{3} \times S^{1}$ in the large $N$ limit. The large $N$ limit is crucial to our analysis, because it allows us to study phase transitions in finite spatial volume. We took advantage of the small $S^{3}$ limit $R \Lambda \rightarrow 0$ to study the partition function using perturbation theory, following Refs. [8-10,32]. The flow of singularities carries information about the appearance of Hagedorn behavior and a confinement/deconfinement phase transition as the graded partition function is deformed into the thermal partition functions of pure Yang-Mills theory.

We observed two basic mechanisms for the transition. In the $n_{f}=1$ theory (SUSY YM) singularities flow in from $q=1$, corresponding to $\beta=0$. For $n_{f}>1$ singularities appear as complex conjugate pairs which collide on the real line and then turn into two real singularities that move apart as $m$ is increased beyond the critical value. These two singularities have opposite residues, so that the associated Hagedorn growth cancels at the critical mass. Note that in either case the critical value of $m_{c} R$ is finite, so that the mechanism which leads to intriguing BoseFermi cancellation and absence of Hagedorn growth in $\tilde{\rho}(E)$ persists for $m R<m_{c} R$. The fact that $m_{c}$ in $n_{f}=2$ theory is larger than the one in the $n_{f}=1$ theory implies that Bose-Fermi cancellation is more robust in the nonsupersymmetric theory than the supersymmetric one under mass deformation.

An obvious question is what happens to the physics we have discussed as $R \Lambda$ is increased from zero. This amounts to studying what happens when the 't Hooft coupling (at the scale $1 / R$ ) is increased. In the case of $\mathcal{N}=4$ super-YangMills theory the complete $\lambda$ dependence of the Hagedorn temperature was studied in Refs. [48,49], while for pure YM theory and nonsupersymmetric theories with adjoint matter, the first corrections in perturbation theory in $\lambda$ were evaluated in Refs. [33,50]. In future work, it may be interesting to leverage these results to quantitatively explore the behavior of singularities in adjoint QCD as a function of $\Lambda R$, but we can already make some qualitative observations.

In this paper, we have interpreted the Hagedorn singularities as being related to deconfinement. This is only possible in the limit $R \Lambda \rightarrow 0$, because (as we already mentioned in the Introduction) the Hagedorn temperature $\beta_{H}$ and the deconfinement temperature $\beta_{d}$ are only equal in the zero-coupling limit $R \Lambda \rightarrow 0$. For generic $R \Lambda, \beta_{d}>\beta_{H}$, and the deconfinement phase transition is first-order. Heuristically this is due to the fact that deconfinement is driven by a change in the sign of the coefficient of $N^{2}$ in the free energy of the quark-gluon plasma as a function of temperature, rather than by the dynamics of the confined phase. As a result, the interpretation of $\beta_{H}$ is that it corresponds to the scale of the spinodal instability of the large $N$ confined phase: the maximal temperature to which the confined phase can be super-heated before local instabilities develop. So at finite $R \Lambda$ a study of the large $N$ complex-temperature plane singularities of the confinedphase partition function remains interesting, but its physical interpretation changes.

A related question concerns the fate of the phase transitions as a function of $m_{c}$ explored in this paper as $R \Lambda$ is increased from zero. We found that in general, $m_{c} \sim$ $1 / R \sim \Lambda /(R \Lambda)$ when $R \Lambda \rightarrow 0$. When $R \Lambda \rightarrow \infty$, it is known that the large $N$ critical value of $m$ is actually $m / \Lambda=0$ in the infinite volume limit [36]. As a result, it is tempting to guess that $m_{c}$ goes to zero monotonically with $1 /(R \Lambda)$. It would be nice to check this hypothesis by an explicit calculation.

\section{ACKNOWLEDGMENTS}

We are grateful to O. Costin for helpful discussions. The work of S. K., T.S. and M. Ü. is supported by the U.S. Department of Energy, Office of Science, Office of Nuclear Physics under Award Number DE-FG02-03ER41260.
[1] T. D. Lee and C. N. Yang, Phys. Rev. 87, 410 (1952).

[2] M. E. Fisher, in Lecture Notes in Theoretical Physics, Vol. 7c, edited by W. E. Brittin (University of Colorado Press, Boulder, 1965).

[3] R. Hagedorn, Nuovo Cimento Suppl. 3, 147 (1965).

[4] T. D. Cohen, J. High Energy Phys. 06 (2010) 098.

[5] T.D. Cohen and V. Krejcirik, J. High Energy Phys. 08 (2011) 138.
[6] N. Cabibbo and G. Parisi, Phys. Lett. 59B, 67 (1975).

[7] L. G. Yaffe, Rev. Mod. Phys. 54, 407 (1982).

[8] B. Sundborg, Nucl. Phys. B573, 349 (2000).

[9] A. M. Polyakov, Int. J. Mod. Phys. A 17, 119 (2002).

[10] O. Aharony, J. Marsano, S. Minwalla, K. Papadodimas, and M. Van Raamsdonk, Adv. Theor. Math. Phys. 8, 603 (2004).

[11] P. Kovtun, M. Unsal, and L. G. Yaffe, J. High Energy Phys. 06 (2007) 019. 
[12] M. Unsal, Phys. Rev. D 80, 065001 (2009).

[13] M. Unsal, Phys. Rev. Lett. 102, 182002 (2009).

[14] M. Unsal and L. G. Yaffe, Phys. Rev. D 78, 065035 (2008).

[15] M. Unsal, Phys. Rev. Lett. 100, 032005 (2008).

[16] P. C. Argyres and M. Unsal, J. High Energy Phys. 08 (2012) 063.

[17] M. M. Anber, E. Poppitz, and T. Sulejmanpasic, Phys. Rev. D 92, 021701 (2015).

[18] M. M. Anber and E. Poppitz, J. High Energy Phys. 10 (2015) 051.

[19] M. M. Anber, S. Collier, E. Poppitz, S. Strimas-Mackey, and B. Teeple, J. High Energy Phys. 11 (2013) 142.

[20] M. M. Anber, E. Poppitz, and B. Teeple, J. High Energy Phys. 09 (2014) 040.

[21] B. Bringoltz and S. R. Sharpe, Phys. Rev. D 80, 065031 (2009).

[22] B. Bringoltz, M. Koren, and S. R. Sharpe, Phys. Rev. D 85, 094504 (2012).

[23] T. Azeyanagi, M. Hanada, M. Unsal, and R. Yacoby, Phys. Rev. D 82, 125013 (2010).

[24] G. Cossu and M. D’Elia, J. High Energy Phys. 07 (2009) 048.

[25] G. Cossu, E. Itou, H. Hatanaka, Y. Hosotani, and J.-I. Noaki, Proc. Sci., LATTICE2013 (2014) 103 [arXiv:1311.0079].

[26] G. Cossu, H. Hatanaka, Y. Hosotani, and J.-I. Noaki, Phys. Rev. D 89, 094509 (2014).

[27] A. Hietanen and R. Narayanan, J. High Energy Phys. 01 (2010) 079.

[28] A. Hietanen and R. Narayanan, Phys. Lett. B 698, 171 (2011).

[29] A. González-Arroyo and M. Okawa, Phys. Rev. D 88, 014514 (2013).

[30] D. J. Gross, R. D. Pisarski, and L. G. Yaffe, Rev. Mod. Phys. 53, 43 (1981).
[31] N. Weiss, Phys. Rev. D 25, 2667 (1982).

[32] T. J. Hollowood and J. C. Myers, J. High Energy Phys. 11 (2009) 008.

[33] O. Aharony, J. Marsano, S. Minwalla, K. Papadodimas, and M. Van Raamsdonk, Phys. Rev. D 71, 125018 (2005).

[34] G. Basar, A. Cherman, D. Dorigoni, and M. Unsal, Phys. Rev. Lett. 111, 121601 (2013).

[35] G. Basar, A. Cherman, and D. A. McGady, J. High Energy Phys. 07 (2015) 016.

[36] A. Cherman, M. Shifman, and M. Ünsal, Phys. Rev. D 99, 105001 (2019).

[37] M. Unsal, Phys. Rev. D 76, 025015 (2007).

[38] G. Basar, A. Cherman, D. A. McGady, and M. Yamazaki, Phys. Rev. Lett. 114, 251604 (2015).

[39] G. Başar, A. Cherman, K. R. Dienes, and D. A. McGady, Phys. Rev. D 92, 105029 (2015).

[40] G. Başar, A. Cherman, K. R. Dienes, and D. A. McGady, J. High Energy Phys. 06 (2016) 148.

[41] O. Costin and M. Huang, Adv. Math. 222, 1370 (2009).

[42] S. Catterall, R. Galvez, and M. Unsal, J. High Energy Phys. 08 (2010) 010.

[43] R. Lohmayer and R. Narayanan, Phys. Rev. D 87, 125024 (2013).

[44] M. García Pérez, A. González-Arroyo, L. Keegan, and M. Okawa, J. High Energy Phys. 08 (2015) 034.

[45] D. Kutasov and N. Seiberg, Nucl. Phys. B358, 600 (1991).

[46] K. R. Dienes, Nucl. Phys. B429, 533 (1994).

[47] K. R. Dienes, M. Moshe, and R. C. Myers, Phys. Rev. Lett. 74, 4767 (1995).

[48] T. Harmark and M. Wilhelm, Phys. Rev. Lett. 120, 071605 (2018).

[49] T. Harmark and M. Wilhelm, Phys. Lett. B 786, 53 (2018).

[50] M. Mussel and R. Yacoby, J. High Energy Phys. 12 (2009) 005 . 